\begin{tabular}{|c|c|}
\hline Title & Dispersion cancellation in high-resolution two-photon interference \\
\hline Author(s) & Okano, Masay uki; Okamoto, Ryo; Tanaka, A kira; Ishida, Shutaro; Nishizawa, Norihiko; Takeuchi, Shigeki \\
\hline Citation & $\begin{array}{l}\text { Physical review. A, 88(4), 043845-1-043845-5 } \\
\text { https://doi.org/10.1103/PhysRevA .88.043845 }\end{array}$ \\
\hline Issue Date & $2013-10-30$ \\
\hline Doc URL & http:/hdl.handle.net/2115/53834 \\
\hline Rights & @2013 A merican Physical Society \\
\hline Type & article \\
\hline File Information & PhysRevA_88_043845.pdf \\
\hline
\end{tabular}

Instructions for use 


\title{
Dispersion cancellation in high-resolution two-photon interference
}

\author{
Masayuki Okano, ${ }^{1,2}$ Ryo Okamoto,,${ }^{1,2}$ Akira Tanaka, ${ }^{1,2}$ Shutaro Ishida, ${ }^{3}$ Norihiko Nishizawa, ${ }^{3}$ and Shigeki Takeuchi ${ }^{1,2, *}$ \\ ${ }^{1}$ Research Institute for Electronic Science, Hokkaido University, Sapporo 001-0020, Japan \\ ${ }^{2}$ The Institute of Scientific and Industrial Research, Osaka University, Osaka 567-0047, Japan \\ ${ }^{3}$ Department of Electrical Engineering and Computer Science, Nagoya University, Nagoya 464-8603, Japan
}

(Received 22 May 2013; published 30 October 2013)

\begin{abstract}
The dispersion cancellation observed in Hong-Ou-Mandel (HOM) interference between frequency-entangled photon pairs has been the basis of quantum optical coherence tomography and quantum clock synchronization. Here we explore the effect of phase dispersion on ultranarrow HOM dips. We show that the higher-order dispersion, the linewidth of the pump laser, and the spectral shape of the parametric fluorescence have a strong effect on the dispersion cancellation in the high-resolution regime with several experimental verifications. Perfect dispersion cancellation with a linewidth of $3 \mu \mathrm{m}$ is also demonstrated through $25 \mathrm{~mm}$ of water.
\end{abstract}

DOI: 10.1103/PhysRevA.88.043845

PACS number(s): 42.50.Ct, 42.65.Lm

\section{INTRODUCTION}

Two-photon interference (TPI) first demonstrated by Hong, $\mathrm{Ou}$, and Mandel (HOM) [1], has become a universal concept in quantum optics. The dispersion cancellation observed in HOM interference between frequency-entangled photon pairs $[2,3]$ is one of the most remarkable phenomena and has been the basis of novel concepts: quantum optical coherence tomography (QOCT) $[4,5]$ and quantum clock synchronization (QCS) [6]. Furthermore, the scientific interest in two-photon interference between frequency-entangled photon pairs is increasing rapidly, for example, in time-frequency entanglement measurement by weak measurements [7] and multimode frequency entanglement [8].

In these concepts and applications, it is critically important to realize high-resolution ("ultranarrow" linewidth) HOM dips against phase dispersion, since the depth resolution of QOCT or the time-synchronization accuracy of QCS is determined by the linewidth of HOM dips. In more detail, the resolution of optical coherence tomography [9] using low-coherence interference (LCI) [10] is highly limited by the group-velocity dispersion (GVD) [11,12]; a resolution of $20 \mu \mathrm{m}$ is typical in ophthalmography without dispersion compensation [13]. Since TPI can achieve better resolution than that of LCI, QOCT is expected to be an alternative to current OCT. However, in the past experimental test of dispersion cancellation, the linewidth of the HOM dip was limited to $19 \mu \mathrm{m}$ [5], which can still be well explained by the theory proposed in 1992 [2,3]. Note that recently the HOM dip with a resolution of approximately $1 \mu \mathrm{m}$ was demonstrated $[14,15]$; however, these experimental demonstrations were without any dispersive media in the optical paths.

The main motivation of this paper is to explore the dispersion cancellation in "ultranarrow" HOM dips. It was conjectured that the nonzero pump-laser linewidth and the higher-order dispersion may limit the dispersion cancellation in the high-resolution TPI; however, these effects have not previously been studied well. Furthermore, in the former studies, there have been discrepancies in the resolution

\footnotetext{
*takeuchi@es.hokudai.ac.jp
}

enhancement factor of TPI to LCI. Some studies obtained a value of 2 [4,5], while others obtained $\sqrt{2}$ [16].

Here we discuss the generalized theory of TPI, which can explain all of these effects and go on to describe the rigorous testing of the theory, namely, the dispersion tolerance of high-resolution TPI. We show that the resolution enhancement factor depends on the spectral shape of the photons, changing from $\sqrt{2}$ for a Gaussian shaped spectrum to 2 for a rectangular one. For the experimental testing of the theory, we constructed a hybrid LCI and TPI setup on which LCI and TPI experiments could be performed using the same parametric fluorescence. We also succeeded in observing the broadening of the TPI signal that occurs due to the nonzero pump-laser linewidth, which can be well explained by the proposed theory. From our results, it is apparent that we achieved "perfect dispersion cancellation" at high resolutions; the resolution of $3.0 \mu \mathrm{m}$ was unchanged even when $25 \mathrm{~mm}$ of water, which corresponds to the diameter of the human eye, was placed in the optical path. In further testing we used 5-mm-thick zinc selenide ( $\mathrm{ZnSe}$ ) plate and observed a strong tolerance; the degradation of the resolution in TPI was tiny (from 3.0 to $3.5 \mu \mathrm{m}$ ), while the resolution of LCI degrades from 3.0 to $410 \mu \mathrm{m}$ in theory. We believe our generalized theory and rigorous experimental proofs are beneficial not only to QOCT and QCS but also to the basic understanding of multiparticle quantum interferences, which are the basis of quantum metrology and quantum information science.

\section{A GENERALIZED THEORY OF TPI}

Here we derive an equation to describe the HOM dips, taking into account the effects of the arbitrary spectral shape of the parametric fluorescence, linewidth of the pump laser, and higher-order dispersion of the media.

Before discussing TPI, we consider LCI. Figure 1(a) shows a scheme of the LCI. The normalized interferogram $I(\tau)$ with a temporal delay $\tau$ between two arms is given by

$$
I(\tau)=1+\operatorname{Re}\left\{e^{-i \omega_{0} \tau} \int d \Omega\left|f_{\mathrm{o}}(\Omega)\right|^{2} e^{-i \Omega \tau+i 2 d \beta(\Omega)}\right\},
$$

where $\Omega$ is the frequency deviation about the central frequency $\omega_{0}, f_{\mathrm{o}}$ is a normalized spectral probability amplitude [17], 

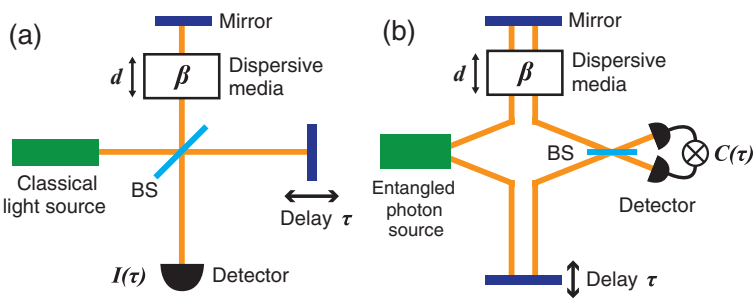

FIG. 1. (Color online) Schematic of LCI (a) and TPI (b). BS stands for beam splitter. $\tau$ is the temporal delay, and $\beta$ is the wave vector in a dispersive media of thickness $d$.

$d$ is the thickness of the media, and $\beta(\Omega)=\beta^{(0)}+\beta^{(1)} \Omega+$ $\frac{1}{2 !} \beta^{(2)} \Omega^{2}+\cdots$ is the wave vector of the light in the dispersive media. $\beta^{(1)}$ is the inverse of the group velocity, and $\beta^{(2)}$ represents the GVD. Here we define the resolution $\Delta L$ by the full width at half maximum (FWHM) of the interferogram. Without any dispersive media, $\Delta L$ is the FWHM $W$ of the Fourier transform of the power spectrum $W\left(\mathrm{FT}\left\{\left|f_{\mathrm{o}}(\Omega)\right|^{2}\right\}\right)$. With a dispersive media, the degraded resolution $\Delta L^{\prime}$ due to the GVD is given by $\Delta L^{\prime}=\Delta L\left[1+\left(2 \sqrt{\ln 2} c \sqrt{\beta^{(2)} d} / \Delta L\right)^{4}\right]^{\frac{1}{2}}$ [17], where $\Delta L$ is the original resolution and $c$ is the speed of light in a vacuum. Thus, $\Delta L^{\prime}$ has a minimum value of $\Delta L$ as seen from $\Delta L_{\mathrm{th}} \sim 2 \sqrt{2 \ln 2} c \sqrt{\beta^{(2)} d}$, which becomes zero when $\beta^{(2)}$ and $d$ are zero, as is the case when no dispersive media is present. The degradation becomes more significant when $\Delta L$ is small (high resolution). For example, the OCT resolution is generally limited to approximately $20 \mu \mathrm{m}$ due to the dispersion of the human eye $\left(\beta^{(2)} d \sim 720 \mathrm{fs}^{2}\right)$ [18].

Next, we discuss the interferograms measured by a TPI setup [Fig. 1(b)]. At first, the biphoton state produced by parametric down-conversion from a source can be described by

$$
|\Psi\rangle=\iint d \omega_{\mathrm{s}} d \omega_{\mathrm{i}} f_{\mathrm{q}}\left(\omega_{\mathrm{s}}, \omega_{\mathrm{i}}\right)\left|\omega_{\mathrm{s}}\right\rangle_{\mathrm{s}}\left|\omega_{\mathrm{i}}\right\rangle_{\mathrm{i}}
$$

where $f_{\mathrm{q}}\left(\omega_{\mathrm{s}}, \omega_{\mathrm{i}}\right)$ is a normalized spectral probability amplitude of the biphoton and $\left|\omega_{\mathrm{s}(\mathrm{i})}\right\rangle_{\mathrm{s}(\mathrm{i})}$ represents the signal (idler) photon state with the frequency $\omega_{\mathrm{s}(\mathrm{i})}$ [19]. The biphoton spectral amplitude $f_{\mathrm{q}}$ can be given by

$$
f_{\mathrm{q}}\left(\omega_{\mathrm{s}}, \omega_{\mathrm{i}}\right)=\xi_{\mathrm{p}}\left(\omega_{\mathrm{s}}, \omega_{\mathrm{i}}\right) f_{\mathrm{s}}\left(\omega_{\mathrm{s}}\right) f_{\mathrm{i}}\left(\omega_{\mathrm{i}}\right)
$$

where $\xi_{\mathrm{p}}\left(\omega_{\mathrm{s}}, \omega_{\mathrm{i}}\right)$ represents the phase-matching condition and $f_{\mathrm{s}(\mathrm{i})}\left(\omega_{\mathrm{s}(\mathrm{i})}\right)$ is a normalized spectral probability amplitude of the signal (idler) photons [20]. The spectrum of each photon of the biphoton is determined by a spatial mode where each photon resides. Then the normalized coincidence count rate $C(\tau)$ of two output modes with a temporal delay $\tau$ is given by

$$
\begin{aligned}
C(\tau)= & 1-\operatorname{Re}\left\{\iint d \omega_{\mathrm{s}} d \omega_{\mathrm{i}} f_{\mathrm{q}}\left(\omega_{\mathrm{s}}, \omega_{\mathrm{i}}\right) f_{\mathrm{q}}^{*}\left(\omega_{\mathrm{i}}, \omega_{\mathrm{s}}\right)\right. \\
& \left.\times e^{i \phi\left(\omega_{\mathrm{s}}, \omega_{\mathrm{i}}, \tau\right)+i \eta\left(\omega_{\mathrm{s}}, \omega_{\mathrm{i}}\right)}\right\}
\end{aligned}
$$

where phase terms $\phi$ and $\eta$ account for the temporal delay $\tau$ and the dispersion $\beta$, respectively. This is the generalized equation of a TPI interferogram, from which the dispersion tolerance at high resolutions can be deduced. Based on this equation, we discuss the resolution enhancement of TPI compared to LCI, the degradation of the resolution due to the nonzero pump linewidth, and the higher-order dispersion effect.

First we consider the resolution enhancement. For simplicity, we consider the case in which a pump laser is monochromatic. For a monochromatic pump with the frequency $\omega_{\mathrm{p}}$, signal and idler photon frequencies are perfectly correlated due to the conservation of the energy $\omega_{\mathrm{p}}=\omega_{\mathrm{s}}+\omega_{\mathrm{i}}$, and the biphoton spectral amplitude is written as

$$
f_{\mathrm{q}}\left(\omega_{\mathrm{s}}, \omega_{\mathrm{i}}\right)=\delta\left(\omega_{\mathrm{p}}-\omega_{\mathrm{s}}-\omega_{\mathrm{i}}\right) f_{\mathrm{s}}\left(\omega_{\mathrm{s}}\right) f_{\mathrm{i}}\left(\omega_{\mathrm{i}}\right),
$$

where $\xi_{\mathrm{p}}$ is a delta function. Substituting Eq. (5) into Eq. (2), the biphoton state is given by

$$
\begin{aligned}
|\Psi\rangle & =\iint d \omega_{\mathrm{s}} d \omega_{\mathrm{i}} \delta\left(\omega_{p}-\omega_{s}-\omega_{i}\right) f_{\mathrm{s}}\left(\omega_{\mathrm{s}}\right) f_{\mathrm{i}}\left(\omega_{\mathrm{i}}\right)\left|\omega_{\mathrm{s}}\right\rangle_{\mathrm{s}}\left|\omega_{\mathrm{i}}\right\rangle_{\mathrm{i}} \\
& =\int d \omega_{\mathrm{s}} f_{\mathrm{s}}\left(\omega_{\mathrm{s}}\right) f_{\mathrm{i}}\left(\omega_{\mathrm{p}}-\omega_{\mathrm{s}}\right)\left|\omega_{\mathrm{s}}\right\rangle_{\mathrm{s}}\left|\omega_{\mathrm{p}}-\omega_{\mathrm{s}}\right\rangle_{\mathrm{i}} \\
& =\int d \Omega f_{\mathrm{s}}(\Omega) f_{\mathrm{i}}(-\Omega)\left|\frac{\omega_{\mathrm{p}}}{2}+\Omega\right\rangle_{\mathrm{s}}\left|\frac{\omega_{\mathrm{p}}}{2}-\Omega\right\rangle_{\mathrm{i}} \\
& \equiv \int d \Omega f_{\mathrm{q}}(\Omega)\left|\frac{\omega_{\mathrm{p}}}{2}+\Omega\right\rangle_{\mathrm{s}}\left|\frac{\omega_{\mathrm{p}}}{2}-\Omega\right\rangle_{\mathrm{i}}
\end{aligned}
$$

where the signal (idler) photon frequency $\omega_{s(i)}$ is $\omega_{p} / 2 \pm \Omega$ with the frequency deviation $\Omega$ and $f_{\mathrm{q}}(\Omega) \equiv f_{\mathrm{s}}(\Omega) f_{\mathrm{i}}(-\Omega)$. Equation (6) is usually used as a description of the entangled biphoton state [3,5]. Then the TPI interferogram in Eq. (4) can be written by

$$
\begin{aligned}
C(\tau) & =1-\operatorname{Re}\left\{\int d \Omega f_{\mathrm{q}}(\Omega) f_{\mathrm{q}}^{*}(-\Omega) e^{-i 2 \Omega \tau}\right\} \\
& =1-\operatorname{Re}\left\{\int d \Omega\left|f_{\mathrm{q}}(\Omega)\right|^{2} e^{-i 2 \Omega \tau}\right\},
\end{aligned}
$$

where $\phi$ is $-2 \Omega \tau$ and $\eta$ is zero. The TPI resolution, which is the FWHM of the interferogram, is half of the FWHM $W$ of the Fourier transform of the spectrum $W\left(\mathrm{FT}\left\{\left|f_{\mathrm{q}}(\Omega)\right|^{2}\right\}\right) / 2$, and the resolution enhancement factor $R_{e}$ is given by

$$
R_{e}=2 \frac{W\left(\mathrm{FT}\left\{\left|f_{\mathrm{o}}(\Omega)\right|^{2}\right\}\right)}{W\left(\mathrm{FT}\left\{\left|f_{\mathrm{s}}(\Omega)\right|^{2}\left|f_{\mathrm{i}}(\Omega)\right|^{2}\right\}\right)} .
$$

Now we discuss $R_{e}$ for the same bandwidth of LCI and TPI $\left(f_{\mathrm{o}}=f_{\mathrm{s}, \mathrm{i}}\right)$. Equation (8) suggests that the resolution enhancement depends on the spectral shape of the photons. $R_{e}$ is 2 for a rectangular shaped spectrum, where $\left|f_{\mathrm{s}}(\Omega)\right|\left|f_{\mathrm{i}}(\Omega)\right|=$ $\left|f_{\mathrm{s}, \mathrm{i}}(\Omega)\right|$. For a Gaussian shaped spectrum, $R_{e}$ reduces to $\sqrt{2}$ because the biphoton spectral width is reduced by $\sqrt{2}$ compared to the single photon spectral width. These results suggest that the discrepancy observed in the previous reports $[4,5,16]$ is due to the difference in the spectral shape of the source used.

Next we consider the dispersion tolerance taking into account a nonzero pump linewidth. When the pump laser has a nonzero linewidth, the biphoton spectrum is given by Eq. (3) and then the TPI interferogram in Eq. (4) with a dispersive media can be written as

$$
\begin{aligned}
C(\tau)= & 1-\operatorname{Re}\left\{\iint d \omega_{\mathrm{s}} d \omega_{\mathrm{i}}\left|\xi_{\mathrm{p}}\left(\omega_{\mathrm{s}}, \omega_{\mathrm{i}}\right)\right|^{2}\left|f_{\mathrm{s}}\left(\omega_{\mathrm{s}}\right)\right|^{2}\left|f_{\mathrm{i}}\left(\omega_{\mathrm{i}}\right)\right|^{2}\right. \\
& \left.\times e^{-i\left(\omega_{\mathrm{s}}-\omega_{\mathrm{i}}\right) \tau+i 2 d\left[\beta\left(\omega_{\mathrm{s}}\right)-\beta\left(\omega_{\mathrm{i}}\right)\right]}\right\},
\end{aligned}
$$


where each photon spectral amplitude is assumed to be the same $\left(f_{\mathrm{s}}=f_{\mathrm{i}}\right)$. For a monochromatic pump, $\eta$ is $4 d \beta^{(1)} \Omega$ and the GVD $\beta^{(2)}$ is completely canceled within the secondorder dispersion approximation, indicating a perfect dispersion tolerance. However, the dispersion tolerance becomes imperfect for a pump with a certain nonzero linewidth. When $\xi_{\mathrm{p}}$ is the Gaussian of $\omega_{\mathrm{p}}=\omega_{\mathrm{s}}+\omega_{\mathrm{i}}$ with the bandwidth $\Delta \omega_{p}$ and $f_{\mathrm{s}(\mathrm{i})}$ is also Gaussian with the bandwidth $\Delta \omega_{s(i)}$, the original resolution $\Delta L$ degrades to $\Delta L^{\prime}=[1+$ $\left.\left(\Delta \omega_{\mathrm{s}, \mathrm{i}} \Delta \omega_{\mathrm{p}} \beta^{(2)} d / 8 \sqrt{2} \ln 2\right)^{2}\right]^{\frac{1}{2}} \Delta L$ [21], where $\beta^{(2)}$ cannot be canceled. The degradation becomes more significant when the pump linewidth is larger.

Finally, we consider the higher-order dispersion effect. For higher-order approximation of dispersion in the media, the interferogram with a monochromatic pump can be written from Eq. (4) as

$$
\begin{aligned}
C(\tau)= & 1-\operatorname{Re}\left\{\int d \Omega\left|f_{\mathrm{q}}(\Omega)\right|^{2}\right. \\
& \left.\times e^{-i 2 \Omega \tau+i 4 d\left(\frac{\beta^{(3)}}{3 !} \Omega^{3}+\cdots\right)}\right\},
\end{aligned}
$$

where the low-order dispersion $\beta^{(1)}$ is omitted. The third-order dispersion $\beta^{(3)}$ is not canceled as all odd-order dispersion. The asymmetric phase dispersion with the cubic dependence $\beta^{(3)} \Omega^{3}$ makes interferograms asymmetric [22]. Though the higher-order dispersion is small in general cases, the contribution is not negligible at high resolutions due to the large frequency component $\Omega$.

\section{THE HYBRID LCI/TPI EXPERIMENTAL SETUP}

To experimentally test the generalized theory for highresolution TPI, we constructed a hybrid experimental setup for both LCI and TPI as illustrated in Fig. 2. The cw pump-laser beam (wavelength, $404 \mathrm{~nm}$; linewidth, $\sim 100 \mathrm{kHz}$; power, $100 \mathrm{~mW}$ ) is focused at a type-I phase-matched $\beta$-bariumborate (BBO) crystal, cut by a long-pass filter and stopped by a damper. Parametric fluorescence occurs as frequencyentangled biphotons are generated with a center wavelength of $808 \mathrm{~nm}$ and a bandwidth of $75 \mathrm{~nm}$. For LCI experiments, photons transferred through a polarization-maintaining fiber from $\mathrm{FC}_{\mathrm{A}}$ serve as classical lights. The optical delay $c \tau$ is determined by a delay mirror $A$ and a dispersive media can be set in front of the other mirror. Interfered light is detected by a single-photon-counting module (SPCM, Perkin Elmer, SPCM-AQRH-14). For the TPI experiments, signal photons

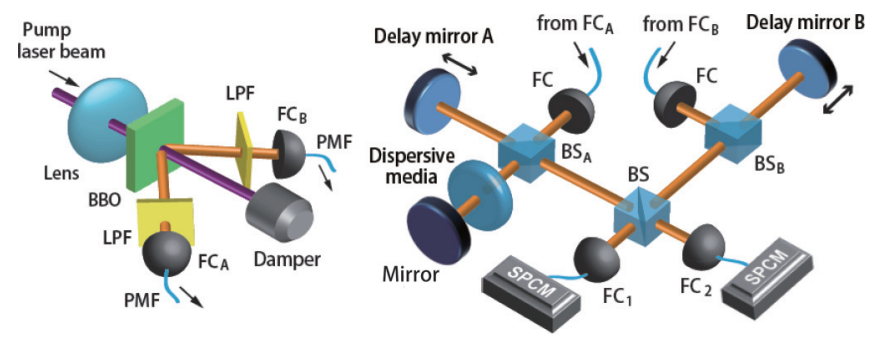

FIG. 2. (Color online) Schematic of the experimental setup, showing the light source (left) and the hybrid LCI/TPI interferometer (right). from the $\mathrm{FC}_{\mathrm{A}}$ travel through the dispersive media, and idler photons from the $\mathrm{FC}_{\mathrm{B}}$ are reflected at the delay mirror $B$ with an optical delay $c \tau$. The coincidence count rate at the $\mathrm{FC}_{1,2}$ is measured by two SPCMs.

\section{RESULTS AND DISCUSSION}

\section{A. Spectral-shape dependence of the resolution enhancement}

First, we verified the theory for the resolution enhancement factor $R_{e}$. For a Gaussian shaped spectrum [Fig. 3(a)], experimental LCI and TPI interferograms show FWHMs of $4.2 \pm 0.1$ [Fig. 3(b)] and 3.0 $\pm 0.1 \mu \mathrm{m}$ [Fig. 3(c)], respectively. Interferograms are expressed in units of the optical delay $c \tau / 2$ to represent the physical displacement of the delay mirrors. We used a Gaussian fit to measure the FWHMs of the interferograms. The obtained $R_{e}$ was $1.4 \pm 0.1$, which is consistent with the theoretical factor of $\sqrt{2}$. We then used a trapezoidal shaped spectrum [Fig. 3(d)] produced by controlling the tilting angle of the BBO crystal. The $R_{e}$ factor of $1.7 \pm 0.1$ obtained from the $3.9 \pm 0.1-\mu \mathrm{m} \mathrm{LCI}$ [Fig. 3(e)] and the 2.3 $\pm 0.1-\mu \mathrm{m}$ TPI [Fig. 3(f)] FWHMs is also fairly consistent with the theoretical value of 1.69 . Thus, the experimental results supported our proposed theory.

\section{B. Perfect dispersion cancellation}

Next we performed perfect dispersion tolerance with a narrowband pump laser. A 25-mm-thick volume of water, which is about the diameter of the human eye, was enclosed by two 1-mm-thick BK7 glass plates and placed as the dispersive media. Dispersion tolerance for a water is important in particular for the QOCT application. LCI resolutions of 3.0 and $4.2 \mu \mathrm{m}$ over the threshold $\left(\Delta L_{\mathrm{th}} \sim 20 \mu \mathrm{m}\right)$ are expected to significantly degrade to 55 and $37 \mu \mathrm{m}$, respectively. Actually, the LCI interferogram broadened to $36.8 \pm 0.4 \mu \mathrm{m}$ [Fig. 4(c)] from the original resolution of $4.2 \mu \mathrm{m}$ [Figs. 3(b) and 4(a)]. In contrast, the TPI interferogram was unchanged with a width of $3.0 \pm 0.1 \mu \mathrm{m}$ [Fig. 4(d)] from the original resolution of $3.0 \mu \mathrm{m}$ [Figs. 3(c) and 4(b)]. Thus, perfect dispersion tolerance has been successfully demonstrated.
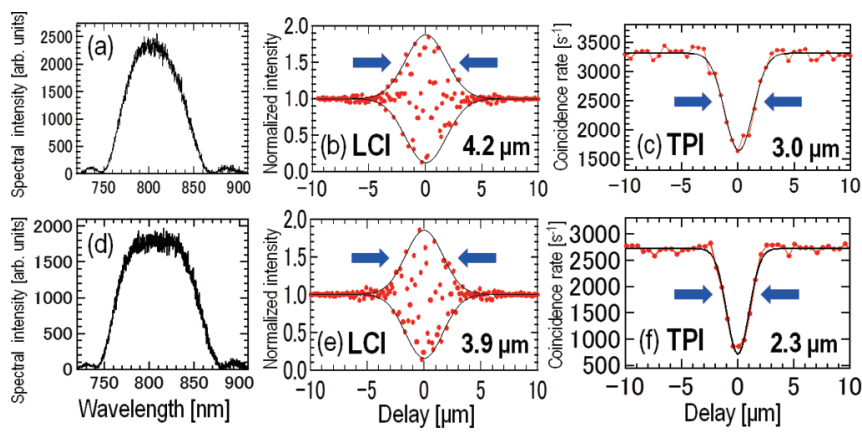

FIG. 3. (Color online) Experimental LCI and TPI interferograms for two spectral shapes: For the Gaussian shaped spectrum (a), the 4.2- $\mu \mathrm{m}$ LCI (b) and the 3.0- $\mu \mathrm{m}$ TPI (c) widths show a resolution enhancement factor $R_{e}$ of 1.4; for the trapezoidal shaped spectrum (d), the 3.9- $\mu \mathrm{m}$ LCI (e) and the 2.3- $\mu \mathrm{m}$ TPI (f) widths show a value of 1.7 for $R_{e}$. The experimental data (red dots) and Gaussian fit curves (black solid line) are plotted. The integration time is $1 \mathrm{~s}$ per point in panels (b), (c), (e), and (f). 


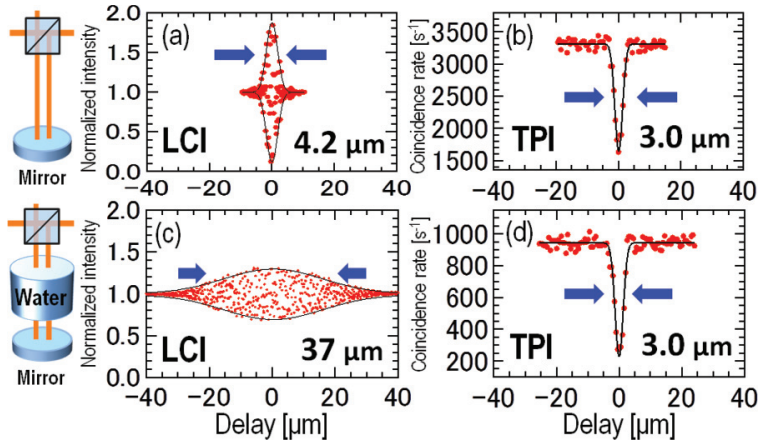

FIG. 4. (Color online) Experimental LCI and TPI interferograms for the Gaussian shaped spectrum: The 4.2- $\mu \mathrm{m} \mathrm{LCI} \mathrm{(a)} \mathrm{and} \mathrm{3.0-}$ $\mu \mathrm{m}$ TPI (b) widths without any media and the broadened $37-\mu \mathrm{m}$ LCI (c) and unchanged 3.0- $\mu \mathrm{m}$ TPI (d) widths using $25 \mathrm{~mm}$ of water as a dispersive media. The integration time is $1 \mathrm{~s}$ per point in panels (a)-(d).

\section{Degradation due to the nonzero pump linewidth}

To test the effect of the nonzero pump linewidth, the $25 \mathrm{~mm}$ of water and a 20-mm-thick BK7 plate were used, which gave a total GVD delay $\beta^{(2)} d$ of $1700 \mathrm{fs}^{2}$. For the narrowband pump laser (linewidth $\sim 100 \mathrm{kHz}$ ), complete dispersion cancellation was achieved with an unchanged FWHM of $3.0 \pm 0.1 \mu \mathrm{m}$ [Fig. 5(a)]. In contrast, for a broadband pump laser (linewidth $\sim 1 \mathrm{THz}$, a laser diode), the FWHM broadened to $3.5 \pm 0.2 \mu \mathrm{m}$ [Fig. 5(b)] with a degradation factor of $1.2 \pm 0.1$. This broadening is close to the theoretical value of 1.17 , meaning our proposed theory has been verified.

\section{Higher-order dispersion effect}

Finally, we tested the higher-order dispersion effect. A 5-mm-thick ZnSe plate $\left(\beta^{(2)} \sim 1000 \mathrm{fs}^{2} / \mathrm{mm}, \beta^{(2)} d \sim\right.$ $5000 \mathrm{fs}^{2}$ [23]) was used as a highly dispersive media. $\beta^{(2)} d$ corresponds to the dispersion caused by $200 \mathrm{~mm}$ of water, which is expected to result in the LCI resolution degrading from 3.0 to $410 \mu \mathrm{m}$. Even with this large GVD, the TPI interferogram maintains a resolution of $3.5-\mu \mathrm{m}$ FWHM by an improvement of over 120 times [Fig. 6(a)]. The tiny degradation can be explained by the third-order dispersion effect. A theoretically simulated TPI interferogram considering the third-order dispersion $\left(\beta^{(3)} \sim\right.$ $\left.870 \mathrm{fs}^{3} / \mathrm{mm}, \beta^{(3)} d \sim 4400 \mathrm{fs}^{3}\right)$ successfully reproduced the experimental result [Fig. 6(b)]. The sign of the asymmetry
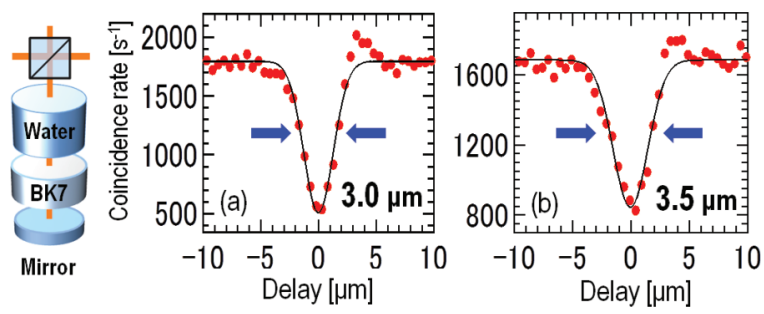

FIG. 5. (Color online) TPI interferograms with dispersive media (25 mm of water and a 20-mm-thick BK7 plate) using narrowband (100 kHz) (a) and broadband (1 THz) (b) pumping-laser linewidths. The nonzero pump linewidth degrades the resolution from 3.0 to $3.5 \mu \mathrm{m}$. The integration time is $1 \mathrm{~s}$ per point in panels (a) and (b).

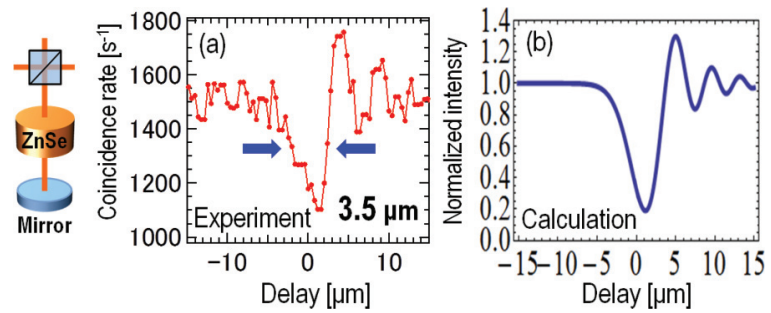

FIG. 6. (Color online) The experimental TPI interferogram with a 5-mm-thick ZnSe plate as a highly dispersive media (a) and a theoretical plot considering the third-order dispersion (b). The integration time is $1 \mathrm{~s}$ per point in panel (a).

is determined by the sign of $\beta^{(3)}$, and the oscillation comes from the high-frequency component $|\Omega|$. A strong dispersion tolerance with a highly dispersive media has been observed, and the higher-order dispersion effect has been demonstrated.

The resolution degradation factors due to the nonzero pump linewidth and the higher-order dispersion are both theoretically estimated to be 1.0 and nonobservable when the resolution of $19 \mu \mathrm{m}[5]$ is assumed. This fact suggests that these effects have become able to be observed due to our high-resolution (3 $\mu \mathrm{m})$ two-photon interference experiment (the factors are 1.2 and 1.3 , respectively, in our experiment.)

It should be also mentioned that the chirped-pulse interferometry [16,24] and the phase-conjugate OCT $[25,26]$ are also promising alternatives to QOCT. In these schemes, the photon flux of the light source can be much higher compared to QOCT, but the realized bandwidth of the correlated light source seems much broader in QOCT [27]. Research using these alternative approaches will have beneficial positive mutual feedback for further investigations.

\section{CONCLUSION}

In conclusion, we investigated the dispersion tolerance of high-resolution HOM interference and clarified all effects of the spectral shape of the source, the linewidth of the pump laser, and the higher-order dispersion using a generalized equation in both theoretical and experimental sides at high resolutions. We have found that the discrepancy in the resolution enhancement factor is due to the difference in the spectral shapes of sources and experimentally verified the spectral dependence. We have also demonstrated perfect dispersion tolerance at $3-\mu \mathrm{m}$ resolution by using a narrowband pump laser and observed a nonperfect dispersion tolerance for a nonzero pump-laser linewidth. We further demonstrated a strong dispersion tolerance in highly dispersive media and observed the higher-order dispersion effect. Our results are important for QOCT and QCS and also offer important insights into quantum information science and technology based on multiparticle quantum interferences.

\section{ACKNOWLEDGMENTS}

This work was supported in part by the Japan Science and Technology Agency CREST project; the Quantum Cybernetics project of the Japan Society for the Promotion of Science (JSPS); the Funding Program for World-Leading Innovative 
R\&D on Science and Technology Program of JSPS; Grantin-Aids from JSPS; the Project for Developing Innovation Systems of the Ministry of Education, Culture, Sports, Science, and Technology; the Network Joint Research Center for
Advanced Materials and Devices; the Research Foundation for Opto-Science and Technology; and the Global COE program. A.T. acknowledges the JSPS Research Fellowship program.
[1] C. K. Hong, Z. Y. Ou, and L. Mandel, Phys. Rev. Lett. 59, 2044 (1987).

[2] A. M. Steinberg, P. G. Kwiat, and R. Y. Chiao, Phys. Rev. Lett. 68, 2421 (1992).

[3] A. M. Steinberg, P. G. Kwiat, and R. Y. Chiao, Phys. Rev. A 45, 6659 (1992).

[4] A. F. Abouraddy, M. B. Nasr, B. E. A. Saleh, A. V. Sergienko, and M. C. Teich, Phys. Rev. A 65, 053817 (2002).

[5] M. B. Nasr, B. E. A. Saleh, A. V. Sergienko, and M. C. Teich, Phys. Rev. Lett. 91, 083601 (2003).

[6] V. Giovannetti, S. Lloyd, L. Maccone, and F. N. C. Wong, Phys. Rev. Lett. 87, 117902 (2001).

[7] H. F. Hofmann and C. Ren, Phys. Rev. A 87, 062109 (2013).

[8] Y. M. Mikhailova, P. A. Volkov, and M. V. Fedorov, Phys. Rev. A 78, 062327 (2008).

[9] D. Huang, E. A. Swanson, C. P. Lin, J. S. Schuman, W. G. Stinson, W. Chang, M. R. Hee, T. Flotte, K. Gregory, C. A. Puliafito, and J. G. Fujimoto, Science 254, 1178 (1991).

[10] M. Born and E. Wolf, Principles of Optics (Cambridge University Press, Cambridge, 1999).

[11] W. Drexler, U. Morgner, F. X. Kärtner, C. Pitris, S. A. Boppart, X. D. Li, E. P. Ippen, and J. G. Fujimoto, Opt. Lett. 24, 1221 (1999).

[12] A. F. Fercher, C. K. Hitzenberger, M. Sticker, and R. Zawadzki, Opt. Express 9, 610 (2001).

[13] C. K. Hitzenberger, A. Baumgartner, W. Drexler, and A. F. Fercher, J Biomed. Opt. 4, 144 (1999).
[14] M. B. Nasr, S. Carrasco, B. E. A. Saleh, A. V. Sergienko, M. C. Teich, J. P. Torres, L. Torner, D. S. Hum, and M. M. Fejer, Phys. Rev. Lett. 100, 183601 (2008).

[15] M. B. Nasr, O. Minaeva, G. N. Goltsman, A. V. Sergienko, B. E. Saleh, and M. C. Teich, Opt. Express 16, 15104 (2008).

[16] J. Lavoie, R. Kaltenbaek, and K. J. Resch, Opt. Express 17, 3818 (2009).

[17] M. Brezinski, Optical Coherence Tomography (Academic, New York, 2006) .

[18] A. G. Van Engen, S. A. Diddams, and T. S. Clement, Appl. Opt. 37, 5679 (1998).

[19] R. A. Campos, B. E. A. Saleh, and M. C. Teich, Phys. Rev. A 42, 4127 (1990).

[20] A. Joobeur, B. E. A. Saleh, and M. C. Teich, Phys. Rev. A 50, 3349 (1994).

[21] K. J. Resch, R. Kaltenbaek, R. Lavoie, and D. N. Biggerstaff, Proc. SPIE 7465, 7465N (2009).

[22] R. Okamoto, S. Takeuchi, and K. Sasaki, Phys. Rev. A 74, 011801 (2006)

[23] Handbook of Optics, 3rd ed., edited by M. Bass, Vol. 4 (McGrawHill, New York, 2009).

[24] R. Kaltenbaek, J. Lavoie, D. N. Biggerstaff, and K. J. Resch, Nat. Phys. 4, 864 (2008)

[25] B. I. Erkmen and J. H. Shapiro, Phys. Rev. A 74, 041601 (2006).

[26] J. Le Gouët, D. Venkatraman, F. N. C. Wong, and J. H. Shapiro, Opt. Lett. 35, 1001 (2010).

[27] A. Tanaka, R. Okamoto, H. H. Lim, S. Subashchandran, M. Okano, L. Zhang, L. Kang, J. Chen, P. Wu, T. Hirohata, S. Kurimura, and S. Takeuchi, Opt. Express 20, 25228 (2012). 